\title{
Acknowledgment to Referees
}

(c) Springer Nature Switzerland AG 2018

Dear Reader,

As we reach the final issue of Drugs \& Aging for 2018, we wish to reflect on another successful year's achievements, and to thank all those who have contributed their time and efforts to guarantee the high quality of our content.

We especially thank the authors who have contributed articles to the journal this year, whose skill and dedication are critical to the continued publication of the journal. The quality of published articles is also testament to the significant efforts of the peer reviewers, whose commitment ensures that the journal's content is held to the highest possible standard. Therefore, on behalf of everyone at the journal, we thank the following individuals who acted as reviewers for Drugs \& Aging in the last 12 months:

\author{
Ahmed H. Abdelhafiz, UK \\ Aza Abdulla, UK \\ Cory Adamson, USA \\ Sri Mahavir Agarwal, Canada \\ Jose Aguirre, Switzerland \\ Kannayiram Alagiakrishnan, Canada \\ Steven M. Albert, USA \\ Emmanuel Andres, France \\ Angelo Antonini, Italy \\ Isabelle Arnet, Switzerland \\ Beatrice Arosio, Italy \\ Anastasios Athanasopoulos, Greece \\ Alireza Atri, USA \\ Ashish Bajel, Australia \\ Arjun V. Balar, USA \\ Capucine Baldini, France \\ Karen Ballen, USA \\ Ian G. Barr, Australia \\ Marisa Battistella, Canada \\ David Beck, USA \\ Steven Belknap, USA \\ Philippe Beuzeboc, France \\ Angela K. Birnbaum, USA \\ J. R. Blanco, Spain \\ Dominick Bosse, USA \\ C. M. Boyd, USA \\ Jess Wallace Brallier, USA \\ Joshua David Brown, USA
}

\author{
W. Timothy Brox, USA \\ Wolfgang Brueckl, Germany \\ Olivier Bruyere, Belgium \\ Julie P. Bynum, USA \\ Arturo Cafolla, Italy \\ Einstein Francisco Camargos, Brazil \\ Noll L. Campbell, USA \\ Jose M. Carrascosa, Spain \\ Ronald Lynel Castelino, Australia \\ Priyanka Chandratre, UK \\ Chirn-Bin Chang, Taiwan \\ V. G. Chel, the Netherlands \\ Jean Chenot, Germany \\ Arrigo F. G. Cicero, Italy \\ Bart L. Clarke, USA \\ Philip G. Conaghan, UK \\ Christopher John Crnich, USA \\ Alfonso J. Cruz-Jentoft, Spain \\ Lori Daiello, USA \\ Kieran Dalton, Ireland \\ Susan R. Davis, Australia \\ Constantinos H. Davos, Greece \\ Nienke A. de Glas, the Netherlands \\ Suzanne M. De La Monte, USA \\ Michel De Pauw, Belgium \\ Navin Kumar Devaraj, Malaysia \\ Jean-Pierre Devogelaer, Belgium \\ G. Dimopoulos, Greece
}


James F. Donohue, USA

Thomas C. Dowling, USA

Katie Druce, UK

Mary Duah-Owusu White, UK

Hang-Korng Ea, France

Cumali Efe, Turkey

T. D. Egan, USA

Abdel El Rafei, USA

Monique Elseviers, Belgium

Akihiro Endo, Japan

Carlos Luis Errando, Spain

María Asunción Esteve-Pastor, Spain

Mahyar Etminan, Canada

Cristian Falup-Pecurariu, Romania

Martin R. Farlow, USA

Barbara Farrell, Canada

Edward Faught, USA

Colin Faulkner, Canada

Aoife Marie Fleming, Ireland

Federica Fogacci, Italy

J. W. Foppe van Mil, the Netherlands

Andrew H. Ford, Australia

Claudio Querido Fortes, Brazil

Susan H. Fox, Canada

Walter Fries, Italy

Martin Früh, Switzerland

Giovanni Gabutti, Italy

Revital Gandelman-Marton, Israel

David M. Gardner, Canada

Pietro Gareri, Italy

Doron Garfinkel, Israel

Beatrice Gasperini, Italy

Serge Gauthier, Canada

Lauren Gerlach, USA

K. Giannitsas, Greece

Danijela Gnjidic, Australia

Steven R. Goldstein, USA

Ana Isabel Gonzalez-Gonzalez, Germany

Sarah J. Goodlin, USA

Allan A. Gottschalk, USA

Isabelle Gouin-Thibault, France

Claudia Felicia Gravina, Brazil

Robert Grenfell, Australia

Nina Griese-Mammen, Germany

Serap Gur, USA

Walter Emil Haefeli, Germany

Serena Halls, UK

Rola N. Hamam, Lebanon

Hideake Hamano, Japan

Benjamin Hyun Han, USA

David A. Hanley, Canada

Yuko Hara, USA

James Haridy, Australia
Anders Daniel Helldén, Sweden

Toby Helliwell, UK

Fredrik Hieronymus, Sweden

Jodie Belinda Hillen, Australia

Robert J. Holt, USA

Ingrid Hopper, USA

Laurence G. Howes, Australia

Carmel M. Hughes, UK

Jose Huguet, Spain

Philippe Huot, Canada

Jacob Hutchins, USA

Kozue Ikeda, Japan

Waguih William IsHak, USA

Stephen H. D. Jackson, UK

Jan G. Jakobsson, Sweden

Slobodan M. Jankovic, Serbia

Michael W. Jann, USA

Jeroen Jaspers Focks, the Netherlands

Efraim Jaul, Israel

Kurt Jellinger, Austria

Vanessa Jordan, New Zealand

Robin L. P. Jump, USA

Alisan Kahraman, Germany

Lisa Kalisch Ellett, Australia

Brian Kaskie, USA

Seymour Katz, USA

Carol A. Kauffman, USA

Gretchen G. Kimmick, USA

Mike Kirby, UK

Julien Kirchgesner, France

Cynthia K. Kirkwood, USA

Roger Knaggs, UK

Wolfgang Korte, Germany

Robert G. Kowalski, USA

Ralph Kupka, the Netherlands

Francesca L. La Frenais, UK

Normand Laperriere, Canada

A. Larocca, Italy

Amanda Leggett, USA

Frederic Liote, France

Enwu Liu, Australia

Matthew C. Lohman, USA

Ansgar W. Lohse, Germany

Catherine J. Lucas, Australia

Giancarlo Lucchetti, Brazil

Alejandro Lucia, Spain

Huub Maas, the Netherlands

Robert MacGinley, Australia

Micha T. Maeder, Switzerland

Howard I. Maibach, USA

Louise Mallet, Canada

Arduino A. Mangoni, Australia

Miriam Manosa, Spain 
Gloria Manso, Spain

Ciro Manzo, Italy

Zachary A. Marcum, USA

Patricia Marino, USA

Sumaiyah Mat, Malaysia

Maria Vittoria Matassini, Italy

Donovan T. Maust, USA

Alexandra Mavroeidi, UK

Paolo Mazzola, Italy

Terrance R. McGovern, USA

Andrew J. McLachlan, Australia

Natalie Medlicott, New Zealand

Roberto Cosimo Melcangi, Italy

Alvaro Mena, Spain

Graydon S.Meneilly, Canada

Kadir Ugur Mert, Turkey

Laura Meyer-Junco, USA

Daniela Moga, USA

Jaime M. Monti, Uruguay

Francesco Montorsi, Italy

João Carlos Araújo Morais, Portugal

Vicki A. Morrison, USA

Janne Kaergaard Mortensen, Denmark

Renato R. P. Munhoz, Canada

Thibault Mura, France

Christiane Muth, Germany

Vasi Naganathan, Australia

Prasad S. Nishtala, New Zealand

Josh Niznik, USA

Samer Noaman, Australia

Kim Notenboom, the Netherlands

Daniel O'Connor, Australia

Matthew O'Connell, UK

Graziano Onder, Italy

Susie Orme, UK

Jelena Osmanovic-Barilar, Croatia

Elias Oussedik, Canada

Amir H. Pakpour, Islamic Republic of Iran

Kyoung Chan Park, Republic of Korea

Carole Parsons, UK

Eliseo Pascual, Spain

Luca Pasina, Italy

Francisco Pasquel, USA

Tejal Patel, Canada

Carlos Pavesio, UK

Claudio Pedone, Italy

David M. Peereboom, USA

Karen Pellegrin, USA

Juan M. Pericas, Spain

Emily P. Peron, USA

Gregory M. Peterson, Australia

Gisèle Pickering, France

JoAnn V. Pinkerton, USA
Athanasios Pipilis, Greece

Nicolas S. Piuzzi, USA

Julius Popp, Switzerland

Koen B. Pouwels, the Netherlands

Marco Proietti, Italy

Loic Raffray, France

Shitao Rao, China

Susanna Rapo-Pylkkö, Finland

L. Rasche, USA

Olivier Rascol, France

Gail B. Rattinger, USA

Emily Reeve, Australia

Suzanne Reeves, UK

M. Cary Reid, USA

Andreas Reimold, USA

Michael E. Reznik, USA

Taeho Greg Rhee, USA

Carlos H. Rojas-Fernandez, Canada

Andrea P. Rossi, Italy

Kathryn Rough, USA

Massimiliano Ruscica, Italy

Sabine Ruths, Norway

Elie Saade, USA

Cheryl Ann Sadowski, Canada

Mohammed Saji Salahudeen, Australia

Elizabeth Sapey, UK

Giselle Sarganas, Germany

Rani A. Sarkis, USA

Gabriela Schmajuk, USA

Robert Schonberger, USA

Ute Schwarz, Canada

Jodi Beth Segal, USA

Michael Graham Serpell, UK

Sepehr Shakib, Australia

Jagannath M. Sherigar, USA

Jonathan Silcock, UK

Caroline Sirois, Canada

Dan Siskind, Australia

Heather S. Snyder, USA

Roy L. Soiza, UK

Annemie Somers, Belgium

Francesca Spagnoli, Italy

Galia Spectre, Israel

Anne Spinewine, Belgium

Sydney Springer, USA

B. Steunenberg, the Netherlands

Claudia Stollberger, Austria

Sergio Adrian Strejilevich, Argentina

Til Stürmer, USA

D. L. Sultzer, USA

Tsutomu Takeuchi, Japan

Rajesh Tampi, USA

Maw Pin Tan, Malaysia 
Cara Tannenbaum, Canada

Tiew-Hwa Katherine Teng, Australia

Mauro Tettamanti, Italy

Avner Thaler, Israel

Petra Thürmann, Germany

Joseph M. Unger, USA

Charlie Upton, USA

Willy Marcos Valencia, USA

Mart A. F .J. van de Laar, the Netherlands

Ana Beatriz Vargas-Santos, Brazil

Giustino Varrassi, Italy

Timo Vesikari, Finland

Kirsten K. Viktil, Norway

Stephan von Haehling, Germany

Nick Vozoris, Canada

Kieran Anthony Walsh, Ireland

Avi Weinbroum, Israel

Juanita Westbury, Australia
Chanaka Wijeratne, Australia

Hugo Wijnen, the Netherlands

Barbara C. Wimmer, Australia

Edward J. Wing, USA

Miles D. Witham, UK

Andy Wolff, Israel

Stephen G. Woodhams, UK

Hans Wouters, the Netherlands

Antoinette Wozniak, USA

Edwina J. Wright, Australia

Jerf W. K. Yeung, Hong Kong

Huifeng Yun, USA

Arnold Geoffrey Zermansky, UK

Helga Zoega, Ireland

Niklas Zojer, Austria

Sytse Zuidema, the Netherlands

Sandra A. Zwijsen, the Netherlands

In addition, we thank the members of the journal's Honorary Editorial Board, who have acted as peer reviewers and authors, and have provided guidance on journal content, policy and processes.

We sincerely hope that you have found the articles published throughout the year to be interesting and informative. The editorial schedule for 2019 is well under way, and we are looking forward to bringing you many more high-quality and authoritative articles over the coming year.

With best wishes,

David Williamson

Editor-in-Chief 